\title{
Über den Flavingehalt der Mikroorganismen \\ Von
}

Seizaburo Yamagutchi, Tokugawa Biol. Inst. und Shoichiro Usami, Hokkaido kaiserl. Univ.

(Mitteilung aus dem Tokugawa biologiscben Institut, Tokyo und dem botan. Institut der Hokkaido kaiserl. Universität, Sapporo)

Eingrgangen am 16. November $193^{8}$

Angesichts der Tatsache, daß das Flavin, das einerseits als Vitamin $B_{2}$ zuwachsfördernde Wirkung auf tierische Organismen ausübt, andererseits als Bestandteil der prosthetischen Gruppen der „gelben Fermente“ an verschiedenen Oxydationsprozessen teilnimmt, erschien es uns von großem Interesse, die Verbreitung und die physiologische Rolle desselben bei verschiedenen Zellen und Geweben näher zu verfolgen. Was die Mikroorganismen anbelangt, so erschienen neuerdings einige Mitteilungen, die sich mit dieser Frage beschäftigen. WARBURG und CHRISTIAN (1933) haben zuerst den Flavingehalt von Bäcker- und Bierhefe, Essig-, Milchsäure- und Buttersäurebakterien vergleichend untersucht und bezüglich der Milchsäurebakterien den Wirkungsmechanismus des gelben Ferments bei der Zellatmung eingehend besprochen. PETT (1935) hat dann bei 7 Arten Hefen und 6 Arten Bakterien die Flavinmenge quantitativ bestimmt und den Einfluß von Sauerstoffbedürfnis, von Zusatz meist zellfremder Substanzen u.s.w. auf die Flavinbildung der Hefe studiert. Kürzlich haben ScHüTz und THEORELL (1938) bei 6 fakultativ anaeroben Bakterienarten festgestellt, daß die Art der dargebotenen Zucker und, wenigstens bei 4 Arten, der Sauerstoffbedarf auf den Gehalt der Kulturen an Flavin fast ohne Einfluß bleibt. In der vorliegenden Arbeit haben wir den Flavingehalt von 2 Hefearten und 15 aeroben bzw. fakultativ anaeroben Bakterien bestimmt und die Rolle des Flavoproteins bei der Sauerstoffatmung der betreffenden Mikroorganismen zur Diskussion gestellt.

\section{Methodisches}

Im allgemeinen wurden die Bakterien auf Pepton-Bouillon-Agar, Bacillus mycoides auf Pepton-Bouillon und Hefearten auf Mohrrübenextrakt-Agar mit 2-proz. Glucosezugabe gezüchtet. Die Agarkultur wurde in PETRI-Schalen (von $20 \mathrm{~cm}$ Durchmesser) oder eigens konstruierten Kulturflaschen und die flüssige Kultur in $200 \mathrm{ccm}$ fassenden ERLENMEYER-Kolben ausgeführt. 
Nach 1- bis 2-tägiger Bebrütung bei $30^{\circ}$ (Hefearten und Mikrokokken bei $25^{\circ}$ ) wurde die Ernte gesammelt, in 1 Liter Leitungswasser suspendiert, auf der Zentrifuge gründlich gewaschen und wieder in ca. $100 \mathrm{ccm}$ destilliertem Wasser aufgeschwemmt. Hierauf wurde die Suspension mit 3 Volumen Methanol versetzt, auf der Schüttelmaschiene bei Zimmertemperatur $\left(20-25^{\circ}\right) 8$ Stunden lang kräftig geschüttelt, im Thermostaten bei $30^{\circ}$ übernacht stehen gelassen und zentrifugiert. Durch diese Behandlung wird das in den Zellen vorhandene Flavin in der Regel größtenteils extrahiert. Die abgeschleuderte Masse wurde nochmals in 200 bis $300 \mathrm{ccm}$ Methanolwasser (3:1) suspendiert, im Thermostaten 20 Stunden lang unter zeitweiliger Schüttelung belassen, dann im Wasserbad 10 Minuten lang auf $65^{\circ}$ erwärmt und nach Abkühlung scharf abzentrifugiert. Die vereinigten Methanolauszüge wurden unter vermindertem Druck bis auf 40 bis $80 \mathrm{ccm}$ eingeengt, ein dabei ausfallender farbloser Niederschlag wurde durch Zentrifugieren entfernt. Die klare gelbe Lösung wurde mit Äther, Petroläther und Chloroform geschüttelt, mit gleichem Volumen n-NaOH versetzt, in einem flachrunden Kolben (von $15 \mathrm{~cm}$ Durchmesser und $3 \mathrm{~cm}$ Schichtdicke) gebracht und unter Luftdurchleitung mit einer 500 WattLampe in einem Abstand von $10 \mathrm{~cm}$ bei $10-12^{\circ}$ bestrahlt. Die bestrahlte Lösung wurde mit $2 \mathrm{n}-\mathrm{HCl}$ kongosauer gemacht und mit 2 Vol. Chloroform dreimal ausgeschüttelt. Hierauf wurde der Farbstoff aus dem Chloroform mit $50 \mathrm{ccm} \mathrm{n} / 10-\mathrm{NaOH}$ herausgeholt, mit 2 Vol. Benzol zweimal geschüttelt, mit $\mathrm{HCl}$ kongosauer gemacht und mit 3 bis $4 \mathrm{Vol}$. Chloroform dreimal ausgeschüttelt. Die verreinigten Chloroformauszüge wurden mit dem überschüssigen destillierten Wasser dreimal gewaschen, das Chloroform bei $30^{\circ}$ verdampft und der Rückstand in bestimmte Menge Chloroform gelöst.

Zur fluorometrischen Bestimmung des entstandenen Lumiflavins wurde das Licht aus einer Quartz-Quecksilber-Lampe benutzt, wobei ein Lichtfilter zwischer der Lampe und den Probierröhren eingeschaltet wurde. Als Vergleichsskala dienten dabei Standardlösungen von folgender Konzentrationsreihe:

$\begin{array}{cccccccccc}\text { Nr. } & 1 & 2 & 3 & 4 & 5 & 6 & 7 & 8 & 9 \\ \gamma \text { Lumiflavin pro ccm } & 4.0 & 3.3 & 2.7 & 2.2 & 1.8 & 1.5 & 1.2 & 1.0 & 0.8\end{array}$

Die Standardlösungen wurden aus Hefe wie folgt dargestellt:

$3 \mathrm{~kg}$ Fleischmanns Bäckerhefe wurden in 5 Liter Leitungswasser suspendiert, auf der Zentrifuge zweimal gewaschen, in 12 Liter Methanolwasser (3:1) suspendiert, im Thermostaten bei $30^{\circ} 20$ Stunden lang stehen gelassen und dann zentrifugiert. Die überstehende gelbe Flüssigkeit (ca. 10 Liter) wurde bei $30^{\circ}$ unter ver- 
mindertem Druck bis auf 1.5 Liter eingeengt, nach Beseitigung des dabei ausfallenden weißen Flöckchens durch Zentrifugieren mit Äther, Petroläther und Chloroform geschüttelt, mit Salzsäure zu 3 Prozenten versetzt, mit Zugabe der Fullererde angerührt und dann zentrifugiert. Das Fullererde-Adsorbat wurde in 1 Liter destilliertem Wasser suspendiert, auf der Zentrifuge dreimal gewaschen und schließlich mit 2 Liter Pyridin-Methanol-Wasser (1:1:3) eluiert. Das Eluat wurde bis auf $500 \mathrm{ccm}$ eingeengt, mit Eis gekühlt, mit $250 \mathrm{ccm}$ kaltem $2 \mathrm{n}-\mathrm{NaOH}$ versetzt und belichtet. Hierauf wurde die Lösung auf kongosaure Reaktion gebracht, mit 2 Vol. Chloroform dreimal ausgeschüttelt und das Chloroform unter vermindertem Druck bis auf $200 \mathrm{ccm}$ verdampft, worauf der Farbstoff durch Ausschütteln mit $50 \mathrm{ccm} \mathrm{n/10-NaOH}$ extrahiert wurde. Die Lösung wurde nach Entfernung des farblosen Begleitstoffs durch Schütteln mit Benzol mit $\mathrm{HCl}$ angesäuert und mit Chloroform dreimal ausgeschüttelt. Nach dreimaliger Wiederholung dieses Verfahrens wurde die alkalische Lösung (50 ccm), nach WARBURG und CHRISTIAN (1933), mit $10 \mathrm{~g} \mathrm{NaCl}$ versetzt und abzentrifugiert, wobei sich aber fast gar kein Flöckchen abschied. Aus der angesäuerten gelben Lösung wurde der Farbstoff mit $100 \mathrm{ccm}$ Chloroform extrahiert und dann mit $300 \mathrm{ccm}$ destilliertem Wasser dreimal gewaschen. Aus der auf diese Weise gewonnenen gereinigten Lumiflavinlösung wurden die Standardlösungen durch entsprechende Verdünnung mit Chloroform hergestellt, wobei der Farbstoffgehalt derselben nach RUDY (1936) mit Hilfe von PULFRICHschem Stufenphotometer mit dem Filter Nr. 47 bestimmt wurde.

\section{Bemerkungen über die quantitative Bestimmung des Flavins}

Es ist schon von einigen Forschern mitgeteilt, daß bei der U̇berführung des Lactoflavins in Lumiflavin durch Bestrahlung ein nicht unbedeutender Verlust an Farbstoff eintreten kann. So z.B. haben Kuhn, WAGNeR-JAUREgG und Kaltschmitt (1934) gezeigt, daß die dabei herbeigeführte Zersetzung des Flavins um 13 bis 58 Prozenten betrug. Auch WARBURg und Christian (1938a) haben kürzlich gefunden, daß die Ausbeute an Lumiflavin bei der alkalischen Photolyse stets 72 Prozenten betrug, gleichgültig ob man dabei von Lactoflavin, von Lactoflavinphosphorsäure oder von Alloxazin-Adenin-Dinucleotid ausgeht. Da diese Zersetzung bekanntlich bei einer bestimmten Belichtungsvorrichtung von der Dauer der Belichtung sowie der Konzentration der belichteten Lösungen bedingt wird, haben wir nun bei konstanter Belichtungsdauer den Einfluß der Verdünnung der 
Versuchslösungen untersucht. Aus $32 \mathrm{~g}$ (Trockengewicht) FLEISCHMANNs Hefe wurden nach dem im vorangehenden Abschnitt beschriebenen Verfahren $82 \mathrm{ccm}$ Flavinlösung dargestellt $(1 \mathrm{ccm}$ Lösung entspricht $0.39 \mathrm{~g}$ Hefe). Diese Lösung wurde mit Wasser wie folgt verdünnt, mit $\mathrm{NaOH}$ versetzt und unter oben erwähnter Versuchsbedingung belichtet; nach 60 Minuten langer Belichtung geht in allen Ansätzen Lumiflavin vollständig ins Chloroform über:

$\begin{array}{crcccc}\begin{array}{c}\text { Versuchs- } \\ \text { nummer }\end{array} & \begin{array}{c}\text { Lactoflavin- } \\ \text { lösung ccm }\end{array} & \begin{array}{c}\text { Dest. Wasser } \\ \text { ccm }\end{array} & \begin{array}{c}\text { n-NaOH } \\ \text { ccm }\end{array} & \gamma \text { Lumiflavin } & \begin{array}{c}\gamma \text { Lumiflavin } \\ \text { pro g Hefe }\end{array} \\ 1 & 20 & 30 & 50 & 45.9 & 5.9 \\ 2 & 10 & 40 & 50 & 20.9 & 5.4 \\ 3 & 5 & 45 & 50 & 10.0 & 5.1 \\ 4 & 2 & 48 & 50 & 5.4 & 5.2\end{array}$

Aus dieser Tabelle geht es hervor, daß die Zersetzung des Farbstoffs bei verdünnter Lösung ziemlich bedeutender als bei konzentrierter ist. Um den Verlust bei der Bestrahlung möglichst gering zu gestalten, haben wir in den nachfolgend beschriebenen Versuchen stets an bestimmten Zeitabschnitten einen aliquoten Teil der Probe herausgenommen und das Fortschreiten der Überführunsreaktion des Flavins vorsichtig kontrolliert.

Andererseits führen die Adsorption an Fullererde und die darauffolgende Elution mit Pyridin-Methanol-Wasser, die wir auch oben bei Herstellung der Standard-Lumiflavinlösungen angewandt haben, zwar eine weitgehende Entfernung des beigemengten Farbstoffs sowie des farblosen Begleitstoffs herbei, jedoch verursacht das Verfahren auch einen nicht vernachlässigbaren Verlust an Menge des Flavins. Bei der quantitativen Bestimmung des Flavingehalts verschiedener Materialien zeigte ELLINGER (1938), daß die Adsorption des Flavins an Fullererde stets mehr oder minder unvollständig verläuft, und ferner daß je vollkommener die Adsorption, umso unvollständiger die Elution ist. Da wir auch unter Anwendung von diesem Verfahren immer zu niedrige Ausbeute an Lumiflavin, die ja z.B. bei der aus FleischmanNs Hefe dargestellten Flavinlösung durchschnittlich nur 40 bis 60 Prozenten betrug, erhalten haben, haben wir bei unseren quantitativen Analysen von Anwendung der AdsorptionsElutions-Methode Abstand genommen.

Bei den quantitativen Bestimmung des Lumiflavins haben wir ferner gefunden, daß der mittels Stufenphotometers direkt gemessene Flavinwert immer viel höher als der durch fluorometrisch gefundene liegt. Z.B. erhielten wir bei einigen Bakterien folgende Ergebnisse: 


\begin{tabular}{l|c|c}
\hline \multirow{2}{*}{ Bakterienarten } & $\gamma$ Lumiflavin pro $\mathrm{g}$ Trockengewicht \\
\cline { 2 - 3 } & photometrisch & fluorometrisch \\
\hline Bacillus pyocyaneus & 27.1 & 14.1 \\
Bacillus subtilis & 5.6 & 4.1 \\
Proteus vulgaris & 21.3 & 13.5
\end{tabular}

Da die aus Bakterien dargestellten Lumiflavinlösungen mehr oder minder gelblicher als gereinigte Standardlösungen aussehen, ist die Möglichkeit vielleicht nicht ausgeschlossen, daß ein nicht fluoreszierender Farbstoff darin beigemengt ist, der im Blau eine nicht unwesentliche Lichtabsorption aufweist und zwar durch das Adsorptions-Elutions-Verfahren bzw. das Aussalzen mit Kochsalz entfernt werden kann. Unten werden wir daher stets nur die Werte angeben, die durch die fluorometrische Methode erhalten sind.

\section{Ergebnisse}

Die Resultate der Flavinbestimmung bei Mikroorganismen sind in der Tabelle 1 dargestellt. Wie es aus dieser Tabelle hervorgeht, liegt der Flavingehalt der 4 Bakterienarten, Micrococcus ochraceus, Mic. citreus, Bacterium coli communior und Bacillus pyocyaneus, in der Höhe von 10-15 $\gamma$ Lumiflavin pro $g$ Trockengewicht. Bei allen anderen Bakterien- und Hefearten ist der Gehalt viel niedriger und liegt stets unter $10 \gamma$; bei Bac. fuorescens albus und Bac. prodigiosus ja unter $1.5 \gamma$

Zum Vergleich sind die Resultate von WARBURG und Christian (1933), von Schütz und Theorell (1938) sowie von Pett (1935) in Tabelle 2 zusammengestellt. Dabei wurde die quantitative Bestimmung von WARBURG und CHRISTIAN sowie von SCHÜTZ und THEORELL durch Lichtabsorptionsmessung der Lumiflavinlösung für die Wellenlänge $445 \mathrm{~m} \mu$ und die von PETT durch die Fluorometrie der Lactoflavin- bzw. Lumiflavinlösung ausgeführt. Bei Bact. coli beträgt der Gehalt an Lumiflavin 18-27 $\gamma$ pro $\mathrm{g}$ nach ScHÜTZ und THEORELL, unter $1 \gamma$ nach PETT und 3-4 $\gamma$ nach unserer Analyse. Bei Proteus vulgaris fanden wir den Wert 9-14 $\gamma$, aber gar kein Flavin nach PETT. PETT sowie wir haben bei Bacillus subtilis einen größenordnungsmäßig übereinstimmenden Zahlenwert erhalten. Der Unterschied zwischen den von verschiedenen Autoren gefundenen Werten, die ja zuweilen sehr bedeutend von einander abweichen, scheint durch die Verschiedenheit der Stämme, der Kulturbedingungen, der Analysenmethoden u.a. verursacht $\mathrm{zu}$ sein. 
Tabelle 1

\begin{tabular}{|c|c|c|c|c|}
\hline Mikroorganismen & $\begin{array}{l}\text { Kulturdauer } \\
\text { (Tage) }\end{array}$ & $\begin{array}{l}\text { Trockenge- } \\
\text { wicht (g) }\end{array}$ & $\begin{array}{l}\text { Lumiflavin } \\
\qquad(\gamma)\end{array}$ & $\begin{array}{l}\gamma \text { Lumiflavin pro } \\
\text { g Trockengew. }\end{array}$ \\
\hline Microc. ochraceus & $\begin{array}{l}2 \\
2\end{array}$ & $\begin{array}{l}2.07 \\
1.58\end{array}$ & $\begin{array}{l}26.8 \\
21.9\end{array}$ & $\begin{array}{l}12.9 \\
13.9\end{array}$ \\
\hline Microc. citreus & $\begin{array}{l}2 \\
2\end{array}$ & $\begin{array}{l}1.38 \\
3.46\end{array}$ & $\begin{array}{l}14.0 \\
31.4\end{array}$ & $\begin{array}{r}10.1 \\
9.1\end{array}$ \\
\hline Microc. agilis & $\begin{array}{l}2 \\
2\end{array}$ & $\begin{array}{l}1.28 \\
1.86\end{array}$ & $\begin{array}{l}2.9 \\
4.9\end{array}$ & $\begin{array}{l}2.3 \\
2.6\end{array}$ \\
\hline WeiBes Sarcina & $\begin{array}{l}2 \\
2\end{array}$ & $\begin{array}{l}3.09 \\
1.60\end{array}$ & $\begin{array}{r}11.1 \\
8.2\end{array}$ & $\begin{array}{l}3.6 \\
5.1\end{array}$ \\
\hline Bac. pyocyaneus & $\begin{array}{l}1 \\
1\end{array}$ & $\begin{array}{l}3.77 \\
1.35\end{array}$ & $\begin{array}{l}53.1 \\
16.3\end{array}$ & $\begin{array}{l}14.1 \\
12.1\end{array}$ \\
\hline $\begin{array}{c}\text { Bac. fuorescens } \\
\text { liquefaciens }\end{array}$ & $\begin{array}{l}1 \\
1\end{array}$ & $\begin{array}{l}1.43 \\
2.46\end{array}$ & $\begin{array}{r}8.2 \\
12.7\end{array}$ & $\begin{array}{l}5.7 \\
5.2\end{array}$ \\
\hline $\begin{array}{l}\text { Bac. fluorescens } \\
\text { albus }\end{array}$ & $\begin{array}{l}2 \\
2\end{array}$ & $\begin{array}{l}1.58 \\
1.38\end{array}$ & $\begin{array}{l}2.3 \\
2.0\end{array}$ & $\begin{array}{l}1.5 \\
1.4\end{array}$ \\
\hline Bac. subtilis & $\begin{array}{l}1 \\
1\end{array}$ & $\begin{array}{l}4.50 \\
3.40\end{array}$ & $\begin{array}{r}18.3 \\
8.4\end{array}$ & $\begin{array}{l}4.1 \\
2.5\end{array}$ \\
\hline $\begin{array}{c}\text { Bac. mesentericus } \\
\text { vulgatus }\end{array}$ & $\begin{array}{l}1 \\
1\end{array}$ & $\begin{array}{l}2.07 \\
1.63\end{array}$ & $\begin{array}{l}8.6 \\
8.4\end{array}$ & $\begin{array}{l}4.2 \\
5.1\end{array}$ \\
\hline Bac. mycoides & $\frac{1}{2}$ & $\begin{array}{l}1.69 \\
1.00\end{array}$ & $\begin{array}{r}10.2 \\
4.4\end{array}$ & $\begin{array}{l}6.0 \\
4.4\end{array}$ \\
\hline Staph. citreus & $\begin{array}{l}2 \\
2\end{array}$ & $\begin{array}{l}1.47 \\
1.36\end{array}$ & $\begin{array}{r}8.7 \\
12.2\end{array}$ & $\begin{array}{l}5.9 \\
9.0\end{array}$ \\
\hline Bac. prodigiosus & $\begin{array}{l}2 \\
2\end{array}$ & $\begin{array}{l}3.37 \\
2.48\end{array}$ & $\begin{array}{l}3.3 \\
1.9\end{array}$ & $\begin{array}{l}0.9 \\
0.8\end{array}$ \\
\hline $\begin{array}{l}\text { Bact. } \text { coli } \\
\text { communior }\end{array}$ & $\begin{array}{l}1 \\
1\end{array}$ & $\begin{array}{l}2.35 \\
2.19\end{array}$ & $\begin{array}{l}7.8 \\
9.3\end{array}$ & $\begin{array}{l}3.3 \\
4.3\end{array}$ \\
\hline Proteus vulgaris & $\begin{array}{l}1 \\
1\end{array}$ & $\begin{array}{l}3.33 \\
1.40\end{array}$ & $\begin{array}{l}45.1 \\
11.9\end{array}$ & $\begin{array}{r}13.5 \\
8.5\end{array}$ \\
\hline Proteus mirabilis & $\begin{array}{l}2 \\
1\end{array}$ & $\begin{array}{l}1.47 \\
1.16\end{array}$ & $\begin{array}{l}11.9 \\
11.3\end{array}$ & $\begin{array}{l}8.1 \\
9.7\end{array}$ \\
\hline Fleischmanns Hefe & - & $\begin{array}{l}7.80 \\
3.34\end{array}$ & $\begin{array}{l}45.9 \\
24.9\end{array}$ & $\begin{array}{l}5.9 \\
7.5\end{array}$ \\
\hline Bäckerhefe & 2 & 2.30 & 7.3 & 3.2 \\
\hline Bierhefe & $\begin{array}{l}2 \\
2\end{array}$ & $\begin{array}{l}7.78 \\
3.93\end{array}$ & $\begin{array}{r}22.6 \\
8.9\end{array}$ & $\begin{array}{l}2.9 \\
2.3\end{array}$ \\
\hline
\end{tabular}


Tabelle 2

\begin{tabular}{|c|c|c|}
\hline Mikroorganismen & $r$ Lumiflavin pro $\mathrm{g}$ & Autoren \\
\hline $\begin{array}{l}\text { Lactobac. delbrueckii } \\
\text { Clost. butyricum } \\
\text { Flavobact. lactis } \\
\text { Bact. acidi lactici } \\
\text { Strept. thermophilus } \\
\text { Staph. aureus } \\
\text { Staph. albus } \\
\text { Bact. coli } \\
\text { ". } \\
\text { Prot. vulgaris } \\
\text { Acetobac. pasteurianum } \\
\text { Bac. aerogenes } \\
\text { Bac. subtilis }\end{array}$ & $\begin{array}{c}77 \\
19-34 \\
91 \\
21-29 \\
29-44 \\
10-37 \\
21-80 \\
0 \\
18-27 \\
<1-0 \\
0 \\
10 \\
6.5 \\
5\end{array}$ & 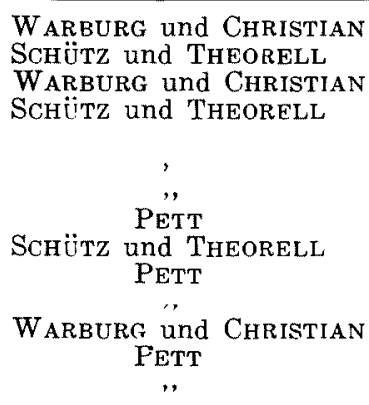 \\
\hline $\begin{array}{l}\text { Bäckerhefe } \\
\quad \text { ". } \\
\text { "top yeast Race M“" } \\
\text { Btop yeast Race XII“ } \\
\text { Bierhefe } \\
\text { „,bottom yeast“" } \\
\text { Torula utilis } \\
\text { Micod. cerevisiae } \\
\text { Pichia membranifaciens }\end{array}$ & $\begin{array}{c}24 \\
17 \\
22 \\
25 \\
20 \\
13-17 \\
18 \\
8 \\
20\end{array}$ & $\begin{array}{l}\text { WARBURG und ChRISTIAN } \\
\text { PETT } \\
\text { WARBURG und ChRISTIAN } \\
\text { PETT } \\
\text { ", } \\
\text { ", }\end{array}$ \\
\hline
\end{tabular}

Die in der Mitteilung von PETT angegehenen Lactoflavinwerte wurden vun uns in die Lumiflavinwerte umgerechntt.

\section{Die Rolle des Flavoproteins bei der Sauerstoffatmung der Mikroorganismen}

Ausgenommen in der Netzhaut befindet sich das Flavin in den Zellen nicht als solches, sondern zum allergrößten Teil in gebundener Form, d.h. als das ,gelbe Ferment“ Was die Mikroorganismen anbelangt so fand z.B. PETT (1935), daß das Flavin in Hefezellen zu ungefähr 81 bis $84 \%$ in nicht dialysierbarer Form vorkommt. Bei den in vitro verlaufenden Dehydrierungen der verschiedenen Zuckerderivaten und organischen Säuren kann man heute mit Recht einsehen, daß das gelbe Ferment die durch Dehydrogenase-SubstratSystem reduzierten Dihydro-Co-Fermente oxydiert, indem es sich in Leucoform verwandelt, welche ihrerseits durch molekulalen Sauerstoff - oder anaerob durch einige Redoxfarbstoffe - wieder in die gelbe Form rückoxydiert wird. Es ist ferner bekannt, daß in vitro die Rückoxydation der reduzierten gelben Ferments nach THEoRELL (1936) durch reines Cytochrom $c$ und nach LAKI (1937) durch das Succinodehydrogenase-Fumarat-System, das weiter mit dem Cytochrom c zu reagieren vermag, glatt hervorgebracht werden kann. Also ist das gelbe Ferment befähigt, sei es direkt oder indirekt ver- 
mittelst cyanhemmbaren Häminsystems mit molekularem Sauerstoff zu reagieren. Wir wollen unten die Rolle des gelben Ferments bei der cyanhemmbaren Atmung und bei der cyanresistenten, d.h. durch Ausschaltung des Häminsystems verlaufenden Atmung gesondert diskutieren.

(1) Cyanhemmbare Atmung. Wir wissen, daß die Sauerstoffatmung von einigen stark atmenden aeroben Mikroorganismen, wie Bäckerhefe und Acetobacter pasteurianum, durch HCN und $\mathrm{CO}$ so gut wie vollständig, dagegen die der meisten von uns oben untersuchten aeroben bzw. fakultativ anaeroben Bakterien dadurch mehr oder minder unvollständig gehemmt wird (vergl. YamaguTcH I 1934). Hierbei ist die Möglichkeit nicht ausgeschlossen, daß die cyanhemmbare Atmung dadurch zustande kommt, daß das durch Dehydrogenase-Coferment-System reduzierte gelbe Ferment auch in vivo wie in vitro unter direkter bzw. indirekter Einwirkung des Häminsystems reoxydiert wird. Aber es sind schon einige Fälle bekannt, wo die gegenseitige Reaktion zwischen Co-Ferment und Häminsystem wohl in Abwesenheit des gelben Ferments vor sich geht. So haben YAKUSHIJI und MoRI (1937) gefunden, daß das von ihnen dargestellte Cytochrom b-Präparat die Fähigkeit besitzt, die Reaktion zwischen Dihydro-Co-Ferment und Cytochrom c direkt zu katalysieren. Andererseits wurde neulich in Cambridge und in Stockholm ein flavinfreies Ferment entdeckt, welches auch die Rückoxydation des Dihydro-Co-Ferments durch das Cytochromsystem bzw. die Redoxfarbstoffe katalysiert, d.h. ,coenzyme factor" von DEWAN und GREEN (1937, 1938) oder „Diaphorase“ von Euler (vergl. ADLER, Euler und Hughes 1938; Euler und Hasse 1938; Euler und HellSTRÖM 1938). Da dieses in tierischen Geweben weit verbreitete Ferment nach EULER und GüNTHER (1938) auch in Hefezellen und nach GREen und DewaN (1938) weiter in Bac. subtilis und Proteus vulgaris und besonders reichlich in Bact. coli vorkommt, so können wir heute darüber nichts sicheres sagen, ob und wie weit die Reaktion zwischen Co-Ferment und Häminsystem in den Zellen durch das gelbe Ferment vermittelt wird oder, mit anderen Worten, in wie weit sich das gelbe Ferment an der cyanhemmbaren Zellatmung beteiligt.

(2) Cyanresistente Atmung. BERTHo und GLÜCK (1932) haben zuerst bei einigen auf anaerobes Leben eingestellten häminlosen Milchsäurebazillen, z.B. Lactobacillus delbrueckii, gefunden, daß die Sauerstoffatmung derselben weder durch HCN noch durch CO gar nicht gehemmt wird. WARBURG und CHRIstian (1933) haben dann gezeigt, daß diese Bakterie besonders viel gelbes Ferment enthält (siehe Tabelle 2), dessen Menge wohl dazu ausreicht, um den 
gesamten bei der Atmung konsumierten Sauerstoff zu übertragen. Derartige direkte Sauerstoffübertragung durch das gelbe Ferment könnte auch bei zwei cytochromführenden Kokkenbazillen, Micrococcus ochraceus und Mic. citreus, wenigstens zum Teil, zur Geltung kommen, da einerseits die Zuckeratmung der genannten Bakterien durch Hämingifte gar nicht gehemmt wird und die sogenannte „Nadi“-Reaktion sehr schwach oder fast ganz negativ ausfällt (vergl. YAMAGUTCHI 1934, 1935), und andererseits der Flavingehalt derselben, wie es oben von uns festgestellt wurde, verhältnismäßig hoch ist. Auch könnte die cyanrefraktäre Atmungsfraktion der üblichen Bakterien vermittelst des gelben Ferments unter Umgehung des vorhandenen Häminsystems stattfinden. Da ferner einige Bakterien die autoxydablen Farbstoffe, wie Pyocyanin in Bacillus pyocyaneus und Prodigiosin in Bacillus prodigiosus, im Zellinnern enthalten, so könnte man sich vorstellen, daß hierbei die cyanunempfindliche Atmung dadurch ermöglicht werde, daß das Dehydrogenase-Co-FermentSystem unter Vermittelung vom gelben Ferment oder vielmehr von Diaphorase den zelleigenen Farbstoff reduziert, der seinerseits direkt mit dem molekularen Sauerstoff in Reaktion geht.

Wie oben diskutiert, kann man sowohl bei der cyanhemmbaren als auch bei der cyanresistenten Atmung recht verschiedene Reaktionswege annehmen. Die Frage, ob und in wie weit dieser oder jener Atmungswege in Zellen verwirklicht werden kann, wird nicht bloß durch die Menge oder Intensität, sondern auch durch die Lokalisation oder Bindungsart der vorhandenen Reaktionsteilnehmer in den Zellen bestimmt. Daher kein Wunder, daß bei den von uns untersuchten Mikroorganismen kein nennenswerter Zusammenhang zwischen der Cyanhemmbarkeit der Atmung und dem Flavingehalt besteht.

An dieser Stelle wollen wir auch darauf aufmerksam machen, daß das durch Lumiflavinreaktion nachgewiesene Flavin in den Zellen der Mikroorganismen nicht immer den Bestandteil der prosthetischen Gruppe des gewöhnlichen gelben Ferments darstellt. Wie kürzlich von WARBURG und CHRISTIAN $(1938 \mathrm{a}, \mathrm{b})$ mitgeteilt, vereinigt sich die Flavinphosphorsäure mit Adenylsäure zum Alloxazin-AdeninDinucleotid, das als ,prosthetische Gruppe“ der in tierischen Geweben und in Hefezellen vorhandenen Aminosäureoxydase fungiert (vergl. Warburg, Christian und Griese 1938). Da nach einer noch nicht veröffentlichten Arbeit von YaMAGUTCHI Alanin durch die oben untersuchten Bakterien fast ausnahmslos rasch oxydiert wird, so sollte ein gewisser Teil des Bakterienflavins offenbar auch als der Bestandteil des WARBURGschen Dinucleotids in den Zellen auftreten. 


\section{Zusammenfassung}

1. Der Flavingehalt von 2 Hefearten und 15 Bakterienarten wurde nach der Lumiflavinreaktion von WARBURG und CHRIsTIAN bestimmt. Der Gehalt liegt bei Micrococcus ochraceus, Mic. citreus, Bacillus pyocyaneus und Bacterium coli communior in der Höhe von 10-15 $\gamma$ Lumiflavin pro $g$ Trockengewicht und bei allen anderen Bakterien- und Hefearten stets unter $10 \gamma$; bei Bac. fuorescens albus und Bac. prodigiosus sogar unter $1.5 \gamma$

2. Einige Fehlerquellen, die bei der quantitativen Bestimmung des Flavins in Betracht kommen, d.h. die Zersetzung des Flavins bei der Belichtung, der Verlust des Flavins beim Adsorptions-ElutionsVerfahren sowie die Verschiedenheit zwischen photometrisch und fluorometrisch bestimmten Flavinwerten, wurden erwähnt.

3. Die Rolle des Flavoproteins bei der cyanhemmbaren bzw. cyanresistenten Zellatmung der Mikroorganismen wurde kurz diskutiert.

Zum SchluB möchten wir gern Herrn Prof. Hon. Dr. K. SHibata, Herrn Prof. Dr. H. HatToRi sowie Herm Prof. Dr. T. Sakamura für liebenswürdige Unterstützung bei dieser Arbeit unseren besten Dank aussagen.

\section{Literatur}

Adler, E., Euler, H. v. und Hughes, w. 1938. Über die Komponenten der Dehydrasesysteme. XVIII. Hoppe-seylers Zeit. f. phys. Chem. 252 : 1-23.

Bertho, B. und Glück, H. 1932. Über den AtmungsprozeB aer Milchsäurebakterien. Liebigs Ann. d. Chem. 494: 159-191.

Dewan, J. G. und Green, D. E. 1937. A new oxidation eatalyst. Nature 140: 10971098.

- 1938. Coenzyme factor-A new oxidation catalyst. Biochem. Journ. 32 : 626-639.

Ellinger, P. 1938. Lyochromes in the kidney. With a note on the quantitative estimation of lyochromes. Biochem. Journ. 32:376-382.

Euler, H. v. und Günther, G. 1938. Diaphorase. Naturwiss. 26: 676-677.

- und Hasse, K. 1938. Diaphorase im Tierkörper. Naturwiss. 26 : 187.

- und Hellström, H. 1938. Zur Kenntnis der enzymatischen Wasserstoffüberträger im Muskel. 2. Mitteilung. Hoppe-Seylers Zeit. f. phys. Chem. 252: 31-40.

Green, D. E. und Dewan, J. G. 1938. Coenzyme factor of yeast. Biochem. Journ. 32: $1200-1203$.

Kuhn, R., Wagner-Jauregg, Th. und Kaltschmitt, H. 1934. Über die Verbreitung der Flavine im Pflanzenreich. Ber. deut. chem. Ges. 67 : 1452-1457.

Laki, K. 1937. Die Oxydation des reduzierten gelben Ferments durch Fumarat. Hoppe-Seylers Zeit. f. phys. Chem. 249: 61-62.

Pett, L. B. 1935. Lactoflavin in micro organisms. Biochem. Journ. 29 : 937-944.

Rudy, H. 1936. Absorptionsspektren im Dienste der Vitaminforschung. Naturwiss. 24 : 497-505.

Schütz, F. und Theorell, H. 1938. Über das gelbe Ferment bei verschiedenen Bakterien. Biochem. Zeit. 295 : 246-251. 
Theorell, H. 1936. Die physiologische Reoxydation des reduzierten gelben Ferments. Biochem. Zeit. 288 : 317-328.

Warburg, O. und Christian, W. 1933. Über das gelbe Ferment und seine Wirkungen. Biochem. Zeit. 266 : 377-411.

- 1938a. Isolierung der prosthetischen Gruppe der d-Aminosäureoxydase. Biochem. Zeit. 298 : 150-168.

- 1938b. Bemerkungen über gelbe Fermente. Biochem. Zeit. 298: 368-377.

-, Christian, W. und Griese, A. 1938. Alloxazin-Adenin-Dinukleotic aus Hefe. Biochem. Zeit. $297: 414$.

Yakushiji, E. und Mori, T. 1987. Untersuchungen über das Cytochrom b. Isolierung, Eigenschaften und seine Rolle im Reaktionsmechanismus der Zellatmung. Acta Phytochim. 10 : 113-123.

Yamagutchi, S. 1934. U̇ber die Beeinflussung der Sauerstoffatmung von verschiedenen Bakterien durch Blausäure und Kohlenoxyd. Beiträge zur Atmungsphysioiogie der Bakterien. I. Acta Pbytochim. 8: 157-172.

- 1935. Untersuchungen über die intrazellulare Indophenolreaktlon bei Bakterien. Beiträge zur Atmungsphysiologie der Bakterien. II. Acta Phytochim. 8: 263-284. 